\title{
VENTRICULAR AND ATRIAL ACTIVITIES ESTIMATION THROUGH SPARSE ECG SIGNAL DECOMPOSITIONS
}

\author{
O. Divorra Escoda, L. Granai, M. Lemay, J. Molinero Hernandez ${ }^{\dagger}$, P. Vandergheynst, J.-M. Vesin \\ Ecole Polytechnique Fédérale de Lausanne, Signal Processing Institute \\ CH-1015 Lausanne, Switzerland, http://itswww.epfl.ch \\ \{lorenzo.granai,mathieu.lemay,pierre.vandergheynst,jean-marc.vesin\}@epfl.ch, \\ oscar.divorra@ieee.org, javiermolinerohernandez@yahoo.es
}

\begin{abstract}
This paper explores a novel approach for ventricular and atrial activities estimation in electrocardiograms (ECG) signals, based on sparse source separation. Sparse decompositions of ECG over signal adapted multi-component dictionaries can lead to natural separation of its components. In this work, dictionaries of functions adapted to ventricular and atrial activities are respectively defined. Then, the weighted orthogonal matching pursuit algorithm is used to unmix the two components of ECG signals. Despite the simplicity of the approach, results are very promising, showing the capacity to generate realistic estimations of atrial and ventricular activity.
\end{abstract}

\section{INTRODUCTION}

Atrial fibrillation (AF) is the most common type of human arrhythmia and it is responsible for about one third of hospitalizations for arrhythmia problems. AF is more frequent in elderly, as its prevalence doubles with each decade of age, from $0.5 \%$ at ages between $50-59$ years to almost $9 \%$ at ages between $80-89$ years. AF is an important clinical entity because of the increased risk of morbidity and mortality. The most frequent consequences are hemodynamic function impairment (loss of atrial synchronized contraction, irregular and inadequately rapid ventricular rate), atriogenic thromboembolic events and tachycardia induced atrial and ventricular cardiomyopathy. AF diagnosis has been assessed for years by visual inspection of the surface electrocardiogram (ECG). On the ECG, the AF signals are characterized by continuous, apparently disorganized, fibrillatory waves (F-waves). Due to the much higher amplitude of the electrical ventricular activity (VA) on the surface ECG, isolation of the atrial activity (AA) component in the ECG is crucial for the study of AF.

Some methods used to solve this problem are based on average beat subtraction. These methods are built on the assumption that the AA is uncoupled with the VA. An average of the ventricular complexes (QRST complexes) is then used to subtract VA [2]. Other methods are based on independent component analysis (ICA). These try to find indepen-

\footnotetext{
${ }^{*}$ We thank Prof. Kappenberger (Lausanne University Hospital, Lausanne Switzerland) for providing the clinical ECG database.

${ }^{\dagger} \mathrm{J}$. Molinero Hernandez was an exchange student from Universitat Politècnica de Catalunya (UPC), Barcelona, Spain. The work presented in this paper was performed in the framework of his M.S. Thesis [1].
}

dent components in an instantaneous linear mixture [3]. A major difficulty in ABS approaches is the limitation imposed by the use of a small number of VA average templates for general VA approximation. In present ICA based approaches, a major gap is that only statistical priors are considered without taking into account the structural nature of signals. In order to circumvent these problems, a possible direction to explore is the use of sparse source separation approaches based on signal adapted redundant dictionaries.

During the past decade, many important advances have been achieved in nonlinear signal approximation methods for sparse decompositions over redundant dictionaries (e.g. [4, $5,6]$ ). In many applications, these techniques offer better performances than those based on orthonormal transforms or direct time domain processing, thanks to their good capacity for efficient signal modeling. In this paper, we present a novel approach for VA and AA estimation. We explore a source separation approach based on sparse decomposition of ECG signals on a redundant multi-component dictionary. Such an approach allows for the consideration of priors on the structural nature of the different class of signals we are willing to separate. The multi-component dictionary is composed by functions specially designed to match the main structural characteristics of VA and AA signals. We also present Weighted Orthogonal Matching Pursuits (Weigthed-OMP) [6] as a tool for generating ECG sparse approximations for source separation.

\section{UNDERDETERMINED SPARSE SOURCE SEPARATION}

Let $f_{\text {mix }}(t): j \in[0, M-1]$ be a set of $M$ signal mixtures generated by the weighted superposition of $N$ source signals $f_{i}(t): i \in[0, N-1]$ such that:

$$
f_{\text {mix }}(t)=\sum_{i=0}^{N-1} a_{j, i} \cdot f_{i}(t)+n_{j}(t),
$$

where $n_{j}(t)$ represents some additive noise.

The problem of source separation is a classical problem in many fields like acoustics, radio or medical signal and image processing. Many applications exist where the retrieval of the different additive components, forming a set of complex signals, is required. The use of sparse signal representations in source separation problems was proposed in [7] in order to 
exploit some prior knowledge about the structural characteristics of each $f_{i}(t)$. Many signals can be sparsely represented by the superposition of a limited number of atoms from an adapted dictionary of functions $\left(\mathcal{D}=\left\{g_{l}(t): l \in \Omega\right\}\right)$ :

$$
f_{i}(t)=\sum_{l \in \Lambda} b_{l}^{i} \cdot g_{l}^{i}(t)+\mathcal{R}_{f_{i}(t)}^{|\Lambda|}
$$

where $b_{l}^{i}$ are the atom coefficients, $\Lambda \subset \Omega$ and $\mathcal{R}_{f_{i}(t)}^{\Lambda}$ is an eventual residual that depends on $\Lambda$.

Combining Eq. (1) and Eq. (2), one derives that, in order to solve the separation problem, the set of mixing coefficients $a_{j, i}$ and a sparse set of expansion coefficients $b_{l}^{i}$ must be recovered while $n_{j}(t)$ are kept as small as possible. Indeed, accurate sparse models can efficiently capture the structural nature of signals, leading to better source separation results as exposed in [7].

A challenging form of sparse source separation problem is when there are fewer mixtures than sources. An underdetermined source separation problem is, for example, to separate the different components from a single ECG channel trace. This is studied in this work through the use of a novel sparse source separation approach. In order to do this, we adapt the two stage separation process proposed in [7] to the particular case of ECG components estimation: First, we a priori design an overcomplete dictionary where sources are assumed to be sparsely representable. Second, the sources are unmixed by exploiting their sparse representability.

\section{ECG COMPONENTS SEPARATION}

In this section, we formulate the ECG activities estimation according to the signal models and sparse source separation strategy described in Sec. 2. The ECG signal $\left(f_{E C G}\right)$ is modeled as a noisy mixture of the two cardiac activities of our interest $\left(f_{A A}\right.$ and $\left.f_{V A}\right)$ :

$$
f_{E C G}=f_{A A}+f_{V A}+n,
$$

where $n$ stands for the noise.

The generation of good sparse models for $f_{A A}$ and $f_{V A}$ requires the use of basis functions fitting their particular structures. As shown in the following, $f_{A A}$ and $f_{V A}$ have quite different characteristics, and this is what enables us to separate them. The approach we propose is based on the decomposition of $f_{E C G}$ on a redundant dictionary $(\mathcal{D})$ composed by the union of two sub-dictionaries: $\mathcal{D}_{V A}$ suited for representing the ventricular activity and $\mathcal{D}_{A A}$, better adapted for representing the atrial activity. In the following $D, D_{V A}$ and $D_{A A}$ stand for the synthesis matrices of $\mathcal{D}, \mathcal{D}_{V A}$ and $\mathcal{D}_{A A}$ respectively, where each column represents an atom of the dictionary. Hence,

$$
f_{E C G} \simeq D \cdot \mathbf{b}=D_{A A} \cdot \mathbf{b}_{A A}+D_{V A} \cdot \mathbf{b}_{V A} .
$$

Given the noisy nature of $f_{E C G}$ and the high complexity of each of its components, in this work, we exclusively consider sparse approximations. According to Eq. (4), b is composed of two parts $\left(\mathbf{b}_{A A}\right.$ and $\mathbf{b}_{V A}$ ), each one containing the coefficients related to $D_{A A}$ and $D_{V A}$. The approach we explore is simple: one generates a sparse approximation of
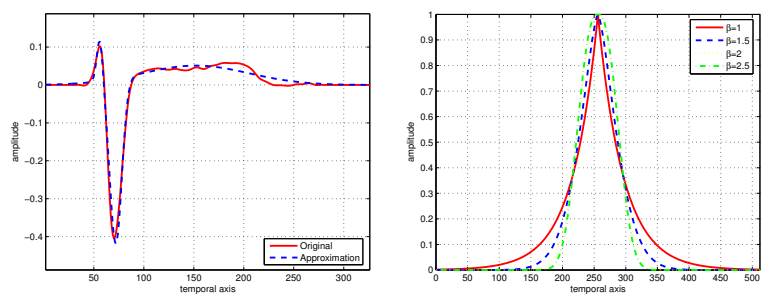

Fig. 1. Left: QRST VA complex and its approximation using just 3 atoms. Right: Effect of $\beta$ on the GGF (see Eq. (5)).

$f_{E C G}$ on $\mathcal{D}$ and then, estimates for $f_{A A}$ and $f_{V A}$ are reconstructed by just using the components from the appropriate dictionary:

$$
f_{A A} \simeq D_{A A} \cdot \mathbf{b}_{A A} \quad \text { and } \quad f_{V A} \simeq D_{V A} \cdot \mathbf{b}_{V A}
$$

\subsection{Modeling ECG Ventricular Activity}

The sub-dictionary $\mathcal{D}_{V A}$ is generated by all possible translations of the Generalized Gaussian Function:

$$
g_{V A}(t)=C_{1} \exp \left(-\left(\frac{|t-p|}{\alpha}\right)^{\beta}\right),
$$

where $C_{1}$ is a normalizing constant, $\alpha$ determines the scale and $\beta$ the peakiness. This waveform allows to well approximate the structure of a VA complex using few atoms. Fig. 1 (left) shows a QRST complex and its approximation by using just 3 atoms. With respect to the simpler Gaussian function, $\beta$ allows us to approximate with more accuracy $\mathrm{Q}$ and $\mathrm{R}$ peaks (see Fig. 1 on the right).

The possible values of $\alpha$ and $\beta$ have been chosen experimentally after an extensive set of tests: $\alpha \in\{3,4, \ldots, 7\} \cup$ $\{49,50, \ldots, 54\}$, the first set adapted for $Q$ and $R$ waves and the second intended for $T$ waves approximation, while $\beta \in$ $\{1.5,1.6, \ldots, 2.2\}$. Together with $p$, this makes $\mathcal{D}_{V A}$ highly coherent, but also very flexible for VA approximation. However, such dictionary is far from being optimal, and several improvements are still possible, mainly concerning the approximation of $\mathrm{T}$ waves.

\subsection{Modeling ECG Atrial Activity}

The sub-dictionary $\mathcal{D}_{A A}$ is generated by all possible translations of a real Gabor function:

$$
g_{A A}(t)=C_{2} \exp \left(-\left(\frac{t-p}{\alpha \sqrt{2}}\right)^{2}\right) \cos \left(\frac{2 \pi k(t-p)}{N}-\Delta \psi\right)
$$

where $C_{2}$ is a normalizing constant, $N$ is the signal length, $\alpha$ tunes the scale, $k$ the frequency and $\Delta \psi$ the phase. This waveform is specially adapted for AA approximation. Indeed, as can be observed in Fig. 2 (left), fibrillating AA is of oscillatory nature, which is a perfect fit for the optimal spatiotemporal frequency localization of Gabor functions (see Fig. 2 on the right).

The values of the Gabor function parameters have been determined through an extensive analysis on several ECG signals (see [1]). During the design of $\mathcal{D}_{A A}$, special care in limiting the maximum correlation between $\mathcal{D}_{A A}$ and $\mathcal{D}_{V A}$ atoms 

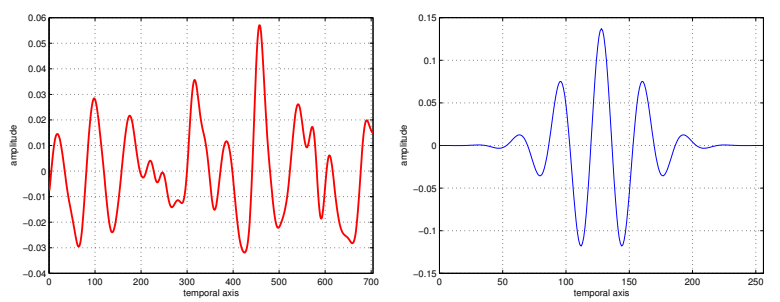

Fig. 2. Left: Example of a simulated AA wave during fibrillation. Right: Gabor atom.

was required. Indeed, excessive coherence between this two sub-dictionaries turns into a complete failure of the algorithm.

\section{ECG SPARSE DECOMPOSITION BY WEIGHTED-OMP}

Eq. (3) involves an underdetermined problem that has no unique solution. The search for the sparsest approximation of $f_{E C G}$ requires the exhaustive test of all coefficient possibilities, i.e. a combinatorial problem. Different alternative approaches have been proposed in order to make computationally affordable the retrieval of a solution for $\mathbf{b}$ (see [4] for a review of some of them). These in general do not guarantee the recovery of the sparsest solution. Nevertheless, recent results show that under certain conditions on the dictionary and the signal, even these fast sub-optimal methods find the sparsest approximation [5].

\subsection{Weighted Orthogonal Matching Pursuit}

Weighted-OMP [6] is used to decompose ECG signals. It is a weighted version of Orthogonal Matching Pursuit (OMP), that iteratively builds $m$-term approximants by selecting at each step the most appropriate term from $\mathcal{D}$ according to a selection rule. Each iteration $k: k \geq 0$ can be seen as a two step procedure:

1. A selection step where an atom $g_{l_{k}} \in \mathcal{D}$ is chosen according to:

$$
g_{l_{k}}=\underset{g_{l} \in \mathcal{D}}{\arg \max }\left|\left\langle r_{k}, g_{l}\right\rangle\right| \cdot w_{l},
$$

where $w_{l} \in[0,1]$ is a pre-estimated weight that reflects, according to a predefined model, the a priori likelihood that atom $g_{l}$ may belong to the good set of atoms best approximating the signal.

2. An orthogonal projection step where an approximant $f_{k+1} \in \operatorname{span}\left(g_{i_{p}}: p \in\{0, \ldots, k\}\right)$, and a residual $r_{k+1}=$ $f-f_{k+1}$ are generated (notice that $r_{0}=f$, and $r_{k+1} \perp$ $\left.f_{k+1} \forall k\right)$. This stage is the one in charge to update, at every step, the set of scalar expansion coefficients.

The signal representation generated by Weighted-OMP is, thus, of the form of Eq. (2).

Weighted-OMP was recently proved in [6] to outperform OMP when using coherent dictionaries and reliable prior information. Weighted-OMP can consider, in the decomposition algorithm, a priori models about the behavior of the dictionary in use with the class of signals to decompose. The use of good enough a priori models can reduce the instability of Weighted-OMP in front of OMP when trying to recover sparse approximations/representations.

\subsection{Weights Generation: Relation Between ECG A Priori Knowledge and the Dictionary}

Thanks to the structure of VA, $f_{E C G}$ can be divided in VA periods. In addition, each VA period can be divided in a set of intervals corresponding to the different VA waves $(\mathrm{Q}$, $\mathrm{R}, \mathrm{S}$ and $\mathrm{T}$ ) and an interval without ventricular activity. VA intervals can be estimated and identified, in practice, through the use of QRST point estimators (e.g. see [8], used in this work). This prior information can thus be used to generate $w_{l} \forall l$. The a priori knowledge obtained from [8] needs to be related with $\mathcal{D}$ in the following way.

$\mathcal{D}$ is divided in $\mathcal{D}_{A A}$ and $\mathcal{D}_{V A}$. According to $f_{E C G}$ nature, AA can be found through all the VA period. Hence, $\mathcal{D}_{A A}$ atoms can not be penalized in terms of this information. This is the reason why in this study we force: $w_{l}=$ $1 \forall l: g_{l} \in \mathcal{D}_{A A}$. To the contrary, the selection of $g_{l} \in \mathcal{D}_{V A}$ can be successfully influenced by the use of the available $a$ priori information. $\mathcal{D}_{V A}$, as seen in Sec. 5 , is composed by a block optimized for QRS waves (ventricular depolarization) and a block designed for $\mathrm{T}$ waves (ventricular repolarization). Depending on the VA interval, $w_{l}$ can be set to 1 for every $g_{l} \in \mathcal{D}_{V A}$ belonging to the appropriate kind for that interval. In case a $g_{l}$ is unsuitable for a given interval, $w_{l}$ can be set to a penalizing factor $0 \leq w_{p}<1$. Thanks to the reliability of the estimators used in this work, it turned out that the best value for $w_{p}$ in our experiments is 0 .

\section{EXPERIMENTAL RESULTS}

\subsection{Validation}

A biophysical computer model of the atria was used to obtain a realistic atrial electrical activity on the torso [9]. The AF signals that were generated in the 12-lead ECG were added to a clinical 4-second standard 12-lead ECG of an AF paroxysmal patient (78 years old) in sinus rhythm in which the $\mathrm{P}$ waves (AA) were removed. The clinical ECG was selected to represent the $\mathrm{VA}$ in $\mathrm{AF}$ as closely as possible. The ratio between the power of the original signal (simulated AA) and the estimation error (estimated AA - simulated AA) was used to evaluate the performance of our method.

\subsection{Results}

First of all, we want to underline that we validated our choice of Weighted-OMP instead of OMP with these simulated measured 4-second ECG signals. By using Weighted-OMP, we increased the SNR in the recovery of VA (respectively, AA) by $0.81 \mathrm{~dB}$ (respectively, $0.65 \mathrm{~dB}$ ). All the following results were obtained by approximating ECG signals with 50 atoms. Fig. 3 shows the resulting separation of VA and AA of the simulated measured 4-second ECG signal on lead V1. One can see how our method succeeds in approximating each one of the VA periods separating, at the same time, the AA with surprising fidelity. In order to study the influence of the AA amplitude on the method, three different simulated AA signals were created; $50 \%, 100 \%$ and $150 \%$ of the original simulated AA amplitude. The ratio between the power of the 


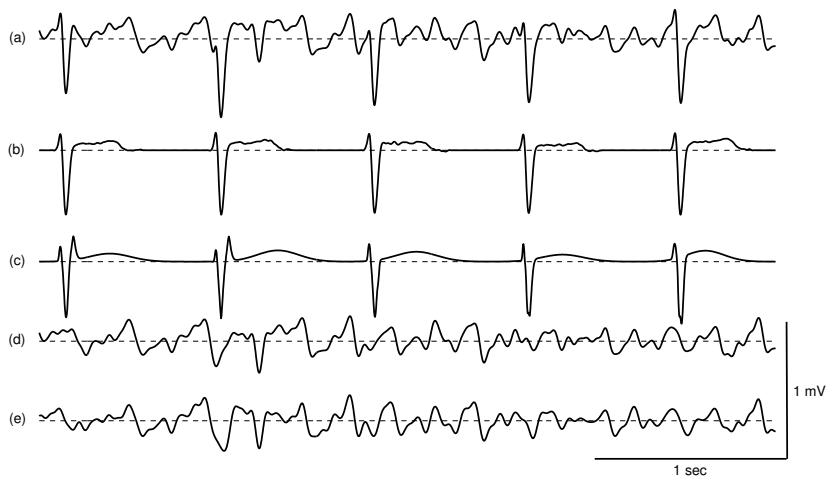

Fig. 3. (a) Simulated measured 4-second ECG signal on V1. (b) Original VA on V1. (c) Estimated VA on V1 (SNR : 8.69 dB). (d) Simulated AA on V1. (e) Estimated AA on V1 (SNR : $6.81 \mathrm{~dB})$.

original activity (VA or AA) and the error on the estimated one was evaluated on leads VR, V1 and V4 (see Table 1). We can observe that the quality of the AA estimation depends on the lead and its original amplitude. The AA SNRs are much higher with the $150 \%$ original amplitude and the overall performance on lead V1 is better than those of other two leads. Of course, the SNR values are directly related to the signal amplitude and in V1, the AA amplitude is higher compared to other leads. However, we observe a decrease of VA estimation performance in lead V1. Fig. 4 shows the resulting separa-

\begin{tabular}{|c||c|c|c|}
\hline \multicolumn{1}{|c||}{} & $0.5 \cdot \mathrm{AA}+\mathrm{VA}$ & $1 \cdot \mathrm{AA}+\mathrm{VA}$ & $1.5 \cdot \mathrm{AA}+\mathrm{VA}$ \\
\cline { 2 - 4 } & \multicolumn{3}{c|}{ lead VR } \\
\hline VA SNR(dB) & 11.06 & 10.88 & 11.08 \\
\hline AA SNR(dB) & -6.94 & -1.05 & 2.61 \\
\hline & \multicolumn{3}{c|}{ lead V1 } \\
\hline VA SNR(dB) & 11.13 & 8.69 & 2.41 \\
\hline AA SNR(dB) & 3.61 & 6.81 & 4.28 \\
\hline & \multicolumn{3}{c|}{ lead V4 } \\
\hline VA SNR(dB) & 12.33 & 11.94 & 11.66 \\
\hline AA SNR(dB) & -6.53 & -0.8 & 2.4 \\
\hline
\end{tabular}

Table 1. Signal-to-noise ratio $(\mathrm{dB})$ on lead VR, V1 and V4. The performance of our method is tested on 3 different AA amplitudes $(50,100$ and $150 \%$ of the original simulated signal).

tion of the VA and AA of the clinical 4-second ECG signal on lead V2.A part from the visually satisfying components separation, these resulting signals were validated using estimated power spectral densities. The dominant frequency of VA (respectively, AA) is between 1 and $2.5 \mathrm{~Hz}$ (respectively, between 3 and $10 \mathrm{~Hz}$ ). The fact that there is no presence of VA dominant frequencies in the AA estimated PSD demonstrates the quality of our clinical results. Further results can be found in [1].

\section{CONCLUSIONS}

In this paper, we present a new framework based on sparse source separation that can be used for QRST cancellation. Results appear to be very promising. Complementary works

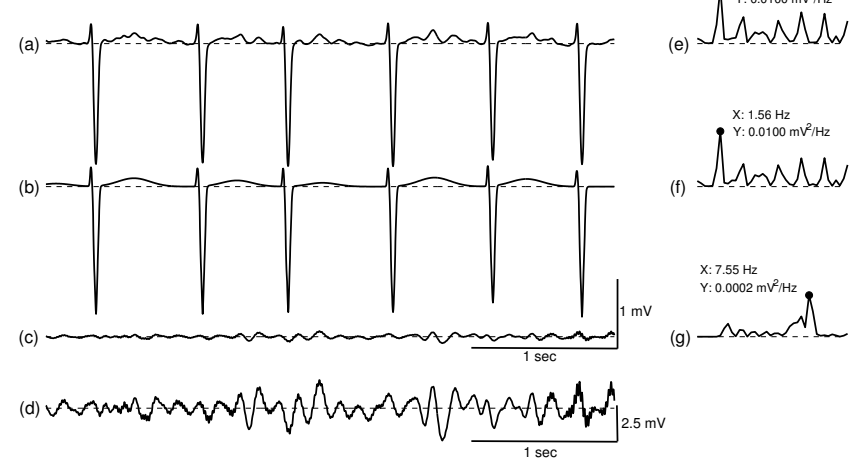

Fig. 4. (a) Clininal 4-second ECG signal on V2 with a dominant frequency of $1.56 \mathrm{~Hz}$ (see its PSD (e)). (b) Estimated VA on V2 with a dominant frequency of $1.56 \mathrm{~Hz}$ (see its PSD (f)). (c) Estimated AA on V2 with a dominant frequency of $7.55 \mathrm{~Hz}$ (see its PSD (g)). (d) Estimated AA on V2 magnified 5 times.

can be spent on the improvement of the dictionary design, specially concerning the $\mathrm{T}$ wave modelling. Moreover, more complex models for a priori knowledge can be studied and the number of atoms used for decomposing signals can be optimized.

\section{REFERENCES}

[1] J. Molinero Hernandez, "Sparse decompositions for ventricular and atrial activity separation," M.S. thesis, Signal Processing Institute, École Polytechnique Fédérale de Lausanne, Switzerland, August 2005.

[2] M. Lemay, V. Jacquemet, A. Forclaz, J.-M. Vesin, and L. Kappenberger, "Spatiotemporal QRST cancellation method using separate QRS and T-waves templates," in Computers in Cardiology 2005. September 2005, IEEE.

[3] F. Castells, J. Igual, J. J. Rieta, C. Sanchez, and J. Millet, "Atrial fibrillation analysis based on ICA including statistical and temporal source information," in ICASSP, 2003, pp. V93-V96.

[4] S. S. Chen, D. L. Donoho, and M. A. Saunders, "Atomic decomposition by basis pursuit," SIAM J. Sci. Comp., vol. 20, no. 1, pp. 33-61, 1999.

[5] D. L. Donoho, M. Elad, and V. Temlyakov, "Stable recovery of sparse overcomplete representations in the presence of noise," IEEE Trans. Inform. Theory, to appear.

[6] O. Divorra Escoda, L. Granai, and P. Vandergheynst, "On the use of a priori information for sparse signal approximations," IEEE Trans. Signal Processing, to appear.

[7] M. Zibulevsky and B. A. Pearlmutter, "Blind source separation by sparse decomposition in a signal dictionary," Neural Computation, vol. 13, no. 4, pp. 863-882, 2001.

[8] R. M. Rangayyan, Biomedical Signal Analysis : A CaseStudy Approach, John Wiley \& Sons, Inc., 2002.

[9] V. Jacquemet, M. Lemay, J.-M. Vesin, A. van Oosterom, and L. Kappenberger, "A biophysical model of ECG signals during atrial fibrillation used to evaluate the performance of QRST cancellation algorithms," in Computers in Cardiology 2005. September 2005, IEEE. 